\title{
Elevated TERT Expression in TERT-Wildtype Adult Diffuse Gliomas: Histological Evaluation with a Novel TERT-Specific Antibody
}

\author{
Kenta Masui, ${ }^{1}$ Takashi Komori ${ }^{D},{ }^{2}$ Yukinari Kato, ${ }^{3}$ Kenkichi Masutomi, ${ }^{4}$ \\ Koichi Ichimura, ${ }^{5}$ Satoshi Ogasawara, ${ }^{3}$ Mika K. Kaneko, ${ }^{3}$ Hiroharu Oki, ${ }^{3}$ \\ Hiroyoshi Suzuki, ${ }^{6}$ Masayuki Nitta, ${ }^{7}$ Takashi Maruyama, ${ }^{7}$ Yoshihiro Muragaki, ${ }^{7}$ \\ Takakazu Kawamata, ${ }^{7}$ Tatsuo Sawada, ${ }^{1}$ and Noriyuki Shibata ${ }^{1}$ \\ ${ }^{1}$ Department of Pathology, Tokyo Women's Medical University, Tokyo 162-8666, Japan \\ ${ }^{2}$ Department of Neuropathology, Tokyo Metropolitan Neurological Hospital, Tokyo 183-0042, Japan \\ ${ }^{3}$ Department of Antibody Drug Development, Tohoku University Graduate School of Medicine, Miyagi 980-8575, Japan \\ ${ }^{4}$ Division of Cancer Stem Cell, National Cancer Center Research Institute, Tokyo 104-0045, Japan \\ ${ }^{5}$ Division of Brain Tumor Translational Research, National Cancer Center Research Institute, Tokyo 104-0045, Japan \\ ${ }^{6}$ Department of Pathology and Laboratory Medicine, Sendai Medical Center, Miyagi 983-0045, Japan \\ ${ }^{7}$ Department of Neurosurgery, Tokyo Women's Medical University, Tokyo 162-8666, Japan
}

Correspondence should be addressed to Takashi Komori; komori-tk@igakuken.or.jp

Received 16 August 2017; Revised 10 December 2017; Accepted 17 January 2018; Published 5 March 2018

Academic Editor: Jens Schittenhelm

Copyright (C) 2018 Kenta Masui et al. This is an open access article distributed under the Creative Commons Attribution License, which permits unrestricted use, distribution, and reproduction in any medium, provided the original work is properly cited.

\begin{abstract}
Telomerase reverse transcriptase (TERT) is important for the biology of diffuse gliomas. TERT promoter mutations are selectively observed among 1p/19q-codeleted oligodendrogliomas and isocitrate dehydrogenase gene- (IDH-) wildtype glioblastoma (GBM). However, TERT transcripts range widely in various cancers including gliomas, and TERT protein expression has been rarely investigated thus far. It would be thus critical to examine the expression level of TERT in tumors in addition to its mutational status, and sensitive and specific methods are urgently needed to examine TERT protein expression for the assessment of TERT biology in gliomas. Using our newly developed TERT-specific monoclonal antibody (TMab-6) applicable to human tissue, we found an unexpected increase in TERT expression in TERT-wildtype as well as TERT-mutated gliomas and in tumor vasculature. This is the first extensive analysis on the expression of TERT immunoreactivity in human glioma tissue, suggesting that TERT protein expression may be regulated by several mechanisms in addition to its promoter mutation.
\end{abstract}

\section{Introduction}

Diffuse gliomas in adults are now separated into three comprehensive tumor groups with distinctive prognoses, based on the status of isocitrate dehydrogenase genes 1 and 2 (IDH1/2) mutations and 1p/19q-codeletion [1]. Recently, hotspot mutations in the telomerase reverse transcriptase gene (TERT) promoter (chromosome 5: 1,295,228 C>T and $1,295,250 \mathrm{C}>\mathrm{T}$ ) have been identified in melanomas [2,3]. The subsequent investigation in a series of gliomas demonstrated the applicability of the TERT promoter mutations in the classification of diffuse gliomas [4], and the selectively high frequency of the TERT promoter mutations was observed among $1 \mathrm{p} / 19 \mathrm{q}$-codeleted oligodendrogliomas and IDHwildtype glioblastoma (GBM) [5]. The presence of TERT promoter mutations is well-correlated with three distinctive glioma groups [1], suggesting that TERT could be deeply involved in the biology of diffuse gliomas.

Hotspot mutations in the TERT promoter (C228T and $\mathrm{C} 250 \mathrm{~T}$ ) are currently recognized as the only event in gliomas to upregulate TERT transcripts, and mRNA of TERT tends to be increased in TERT-mutated gliomas compared with normal brain tissue or TERT-wildtype gliomas [2, 3, 6]. However, the TERT transcripts range widely in expression 
in various cancers including gliomas [5, 7], and there may be other potential mechanisms to upregulate TERT mRNA in addition to its promoter mutations. Indeed, in other brain tumors and systemic cancers, epigenetic shifts in the TERT promoter regions including DNA methylation and histone modifications were reported to regulate TERT transcripts and reactivation [8-10], necessitating a rethinking of the mechanism to upregulate the expression of TERT.

As an essential component of telomerase, TERT adds telomeric repeats to chromosomal ends using telomerase RNA component (TERC) as a template and maintains telomere length. Telomerase activity had been considered to be maintained in most types of the cancer cells, enabling them to proliferate and survive indefinitely [11]. However, a recent report demonstrated that telomere length is positively correlated with mutations in the alpha thalassemia/mental retardation syndrome X-linked gene (ATRX) but not the TERT promoter, suggesting that TERT upregulation itself may be essential for glioma biology irrespective of telomere length [12]. It would be thus critical to examine the expression level of TERT protein in diffuse gliomas to clarify the role of TERT in their biology in addition to its mRNA level as well as mutational status, but few reports have so far validated the expression of TERT protein in human glioma tissue [7]. Sensitive and specific methods are strongly desired to examine TERT protein expression for the future analyses on the TERT biology in diffuse gliomas.

In this study, we developed a TERT-specific antibody applicable to human tissue and examined the expression of TERT in a series of glioma samples. Although the practical diagnostic utility of the antibody to detect the TERT-mutated tumors was expected, TERT immunohistochemistry was not capable of identifying the differences between TERTmutated gliomas and TERT-nonmutant gliomas. Surprisingly, however, an increase in TERT protein expression was detected across all types of gliomas including tumors with wildtype TERT, and the level of TERT expression was different even among the TERT-mutant gliomas such as oligodendrogliomas and GBMs. Our study is the first thorough analysis on the expression of TERT protein using a specific TERT monoclonal antibody in human glioma tissue, suggesting that TERT protein expression may be regulated by several mechanisms in addition to its promoter mutation.

\section{Materials and Methods}

2.1. Tissue Collection. A total of 41 human glioma tissues and 4 nonneoplastic adult human cerebral tissues from four epileptic patients were obtained at surgery from Tokyo Women's Medical University Hospital and Tokyo Metropolitan Neurological Hospital. The tumor samples included 11 grade II and III astrocytic tumors with IDH mutations [diffuse astrocytoma (DA) and anaplastic astrocytoma (AA)], 10 grade II and III oligodendroglial tumors, 11 grade IV $I D H$-wildtype GBMs, and 9 IDH-wildtype diffuse astrocytomas including tumors histologically diagnosed as diffuse astrocytoma, anaplastic astrocytoma, oligoastrocytoma, and anaplastic oligoastrocytoma which did not show IDH mutations, p53 immunopositivity, and the loss of ATRX expression
(wtIDH/p53-/ATRX+). Clinical information of the cases was collected from the medical records. All specimens were fixed in $10 \%$ buffered formalin and embedded into paraffin sections. Sections were routinely stained with hematoxylin and eosin (H\&E), and histopathological diagnoses were made according to the World Health Organization (WHO) 2016 classification of tumors of the nervous system [13-15] and International Society of Neuropathology-Haarlem consensus guidelines [16].

All tumor and control brain samples were subjected to histological diagnosis followed by molecular genetics screening for a more precise/molecular diagnosis (Figure 1(a)) [17]. Tumors in the astrocytoma group (DA/AA) were characterized by detectable immunoreactivity for mutated IDH1 $(\mathrm{R} 132 \mathrm{H})$ and $\mathrm{p} 53$ [18] and the loss of immunoreactivity for ATRX $[19,20]$. All tumors in the oligodendroglioma group demonstrated mutated IDH1 (R132H) and 1p/19q-codeletion. GBM tumors did not possess mutations in $I D H$, corresponding to "GBM, IDH-wildtype" $[14,21]$. The tumors in the $I D H$-wildtype diffuse astrocytoma group did not show any molecular genetic aberrations shown above. The study was approved by the institutional review boards, and the detailed information of the cases was provided in Supplementary Table 1.

\subsection{DNA Extraction and Sanger Sequencing. Genomic DNA} was extracted from formalin-fixed paraffin-embedded (FFPE) tissue of the solid tumor regions confirmed on H\&E sections, using QIAamp DNA FFPE Tissue Kit (QIAGEN, Venlo, The Netherlands), followed by the purification with NucleoSpin gDNA Clean-up Kit (MACHEREY-NAGEL, Dusseldorf, Germany), according to the manufacturer's instructions. Sequences of the primer sets for polymerase chain reaction (PCR) were provided in Supplementary Table 2. PCR was performed using AmpliTaq Gold 360 DNA Polymerase and GC enhancer (Thermo Fisher Scientific, Waltham, MA). Sequencing was carried out by Eurofins Genomics (Tokyo, Japan) with each forward PCR primer as a sequencing primer.

2.3. Molecular Diagnostics. The status of IDH1 (R132H), p53, and ATRX was analyzed by immunohistochemistry using the automated immunostaining processor Histostainer (Nichirei Biosciences, Tokyo, Japan). The antibodies used include antiIDH1 (R132H) (DIA-H09; Dianova, Hamburg, Germany), anti-p53 (DO-7; Nichirei), and anti-ATRX (HPA001906; Sigma-Aldrich, St. Louis, MO). We judged the tumor as p53positive when $>50 \%$ of the tumor nuclei displayed the strong immunoreactivity of $\mathrm{p} 53$, which could be corroborated by the loss of immunoreactivity for ATRX (inactivating mutation) and $1 \mathrm{p} / 19 \mathrm{q}$-nondeletion in the astrocytic tumors [19, 20, 22]. The status of chromosomes $1 p$ and $19 q$ was examined by fluorescence in situ hybridization (FISH) with 1p36 and 19q13 probes (Vysis; Abbott Molecular, Abbott Park, IL), and signal ratios were calculated according to the previous report [23]. Two reported hot spot mutations (C228T and C250T) in a TERT promoter region were analyzed by Sanger sequencing. The hotspot mutations at codon 132 of IDH1 and codon 172 of $\mathrm{IDH} 2$ were also screened by Sanger sequencing if the cases 

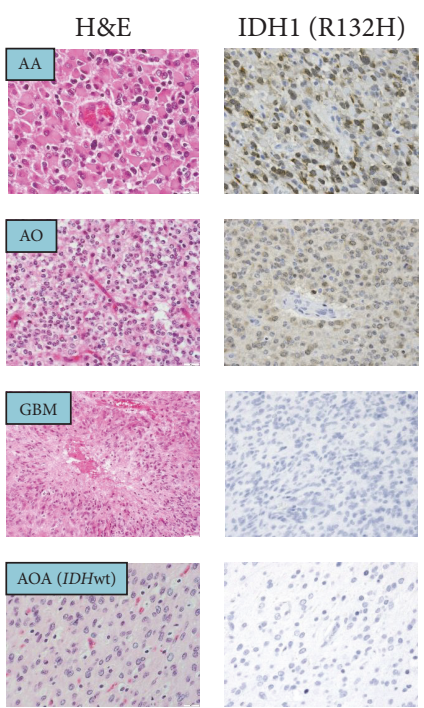

GBM_1 (IDHwt GBM)

C C C C C T C C G G
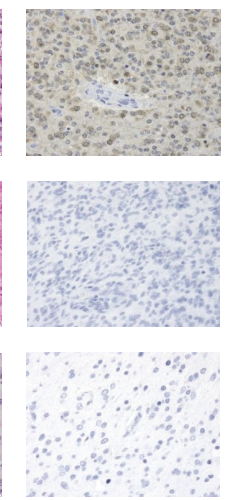

Astro_1 (AA) p53
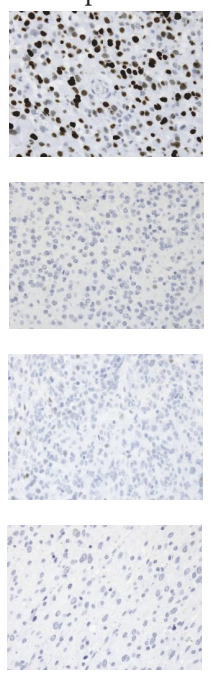

(a)
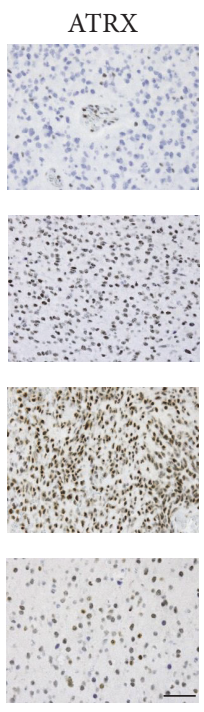

GBM_3 (IDHwt GBM)

C C C C C T C C G G C C C C T C C C G G
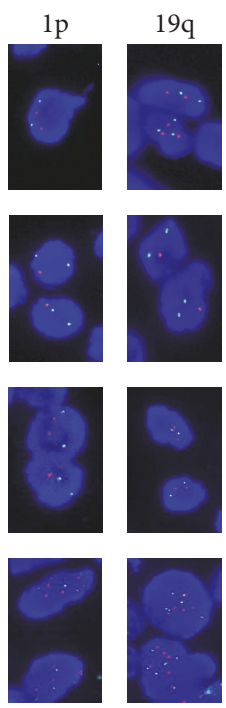

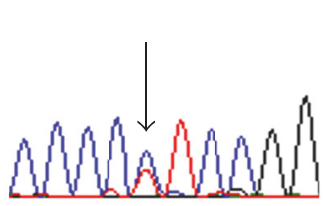

TERT mutation $(\mathrm{C} 228 \mathrm{~T})$

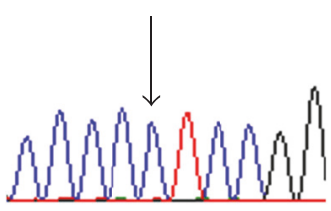

TERT wildtype

(C228)

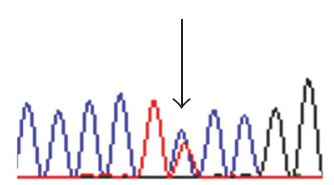

TERT mutation (C250T)
Astro_1 (AA)

C C C C T C C C G G G

(b)

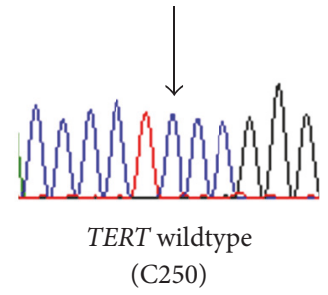

TERT promoter mutations

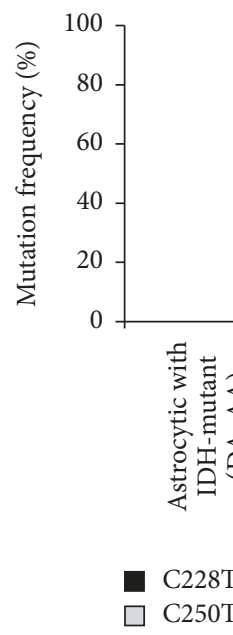

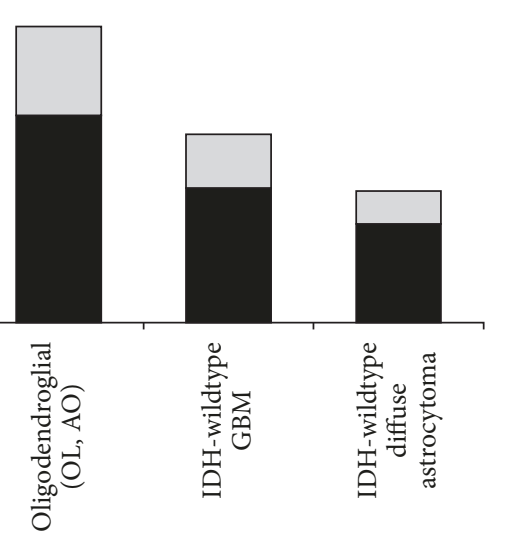

(c)

\author{
GBM_2 (IDHwt GBM) \\ C G C G C T C C C C A \\ Oligo_1 (OL) \\ CGC G C T TC CCA C
}

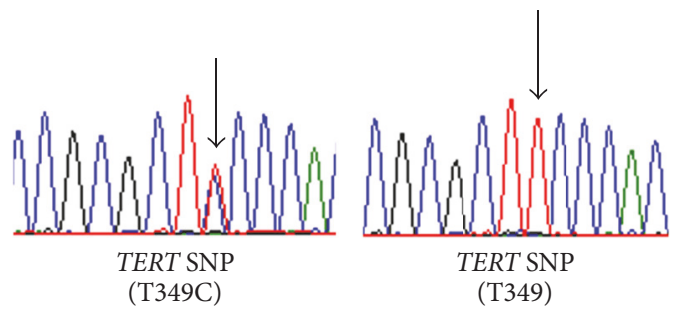

(d)

Figure 1: Molecular profile of the glioma cases. (a) Diffuse astrocytic (DA/AA) tumors were characterized by immunopositivity for IDH1 $(\mathrm{R} 132 \mathrm{H})$ and $\mathrm{p} 53$, and the loss of immunoreactivity for ATRX. Oligodendroglial tumors demonstrated IDH1 (R132H) immunoreactivity and 1p/19q-codeletion on FISH (red: probes for 1p36 or 19q13, green: probes for the centromere of chromosome 1 or 19). Note that these genotypes of astrocytomas and oligodendrogliomas are mutually exclusive except for $I D H$ genes. $I D H$-wildtype GBM tumors did not possess mutations in $I D H$ genes. The $I D H$-wildtype diffuse astrocytoma group did not show any molecular genetic aberrations shown above. (b) Mutations at C228 and C250 of the TERT promoter region were examined for every case by Sanger sequencing. Representative cases were shown for TERT promoter mutations and wildtype TERT. (c) Examination of the frequency of TERT promoter mutations in each group of gliomas. Oligodendroglial tumors and IDH-wildtype GBM possessed high incidence of TERT mutations, followed by the IDH-wildtype diffuse astrocytoma. No grade II and III astrocytic tumors with IDH-mutant showed TERT promoter hotspot mutations. (d) Sanger sequencing results displayed representative cases for TERT promoter common SNPs (T349C). The molecular profile of the cases was summarized in Table 1 and Supplementary Table 1. AA, anaplastic astrocytoma; AO, anaplastic oligodendroglioma; AOA, anaplastic oligoastrocytoma; DA, diffuse astrocytoma; GBM, IDH-wildtype glioblastoma; OL, oligodendroglioma; wt, wildtype. Scale bar $=40 \mu \mathrm{m}$. 
did not show immunoreactivity for mutated IDH1 (R132H) $[24,25]$.

2.4. Cell Lines. U87 malignant glioma cell lines are a kind gift from Dr. Paul Mischel laboratory (Ludwig Institute for Cancer Research, San Diego). Cells were cultured in DMEM supplemented with 10\% FBS (Omega Scientific, Tarzana, $\mathrm{CA}$ ) in a humidified $5 \% \mathrm{CO} 2$ incubator at $37^{\circ} \mathrm{C}$. Analyses with immunocytochemistry and Sanger sequencing were performed using the cell lines as described below.

2.5. Development of TERT-Specific Antibody. Human TERT (hTERT) cDNA (Accession number NM_001193376.1) encoding a specific peptide composed of Glu281-Ala436 amino acid residues was obtained by PCR using a cDNA derived from the LC-AI or HT1080 cell line as a template. The primer set for hTERT was as follows: $5^{\prime}$-AAGGATTTCAGAATTCGAAGCCACCTCTTTGGA-3' (forward) and $5^{\prime}$-TGCCGTCTCCGAATTCCGCCACAGAGCCCTGGG$3^{\prime}$ (reverse). The hTERT-specific peptide was subcloned into an expression vector, pMAL-c2 (New England Biolabs, Beverly, MA) with MAP tag (GDGMVPPGIEDK) [26], and PA tag (GVAMPGAEDDVV) [27] using In-Fusion PCR cloning kit. Competent Escherichia coli (E. coli) TOP-10 cells (Thermo Fisher Scientific) were transformed with the plasmid, pMALc2MAPhPAter/TERTepi. Then, they were cultured overnight at $37^{\circ} \mathrm{C}$ in $\mathrm{LB}$ medium (Thermo Fisher Scientific) containing $100 \mu \mathrm{g} / \mathrm{ml}$ ampicillin (Sigma-Aldrich, St. Louis, MO). Cell pellets were resuspended in phosphate-buffered saline (PBS; nacalai tesque, Kyoto, Japan) with $1 \%$ Triton X-100 with $50 \mu \mathrm{g} / \mathrm{ml}$ aprotinin (Sigma-Aldrich). After sonication, the crude extracts were collected by centrifugation $(9000 \times \mathrm{g}$, $30 \mathrm{~min}, 4^{\circ} \mathrm{C}$ ). The supernatants were loaded onto Amylose resin. The loaded resins were washed extensively with column buffer consisting of $20 \mathrm{mM}$ Tris- $\mathrm{HCl}$ (pH 7.4), $200 \mathrm{mM} \mathrm{NaCl}$ and $1 \mathrm{mM}$ EDTA, and the fusion proteins were eluted by column buffer with $10 \mathrm{mM}$ maltose.

$\mathrm{BALB} / \mathrm{c}$ mice were immunized by intraperitoneal (i.p.) injection of the synthetic peptide of hTERT (302-321 amino acids; Sigma-Aldrich) together with Imject Alum (Thermo Fisher Scientific). After several additional immunizations, a booster injection was given i.p. two days before splenocytes were harvested. The splenocytes were fused with P3U1 cells obtained from the American Type Culture Collection (ATCC; Manassas, VA) using PEG1500 (Roche Diagnostics, Indianapolis, IN). The hybridoma cells were grown in Roswell Park Memorial Institute (RPMI) medium with hypoxanthine, aminopterin, and thymidine selection medium supplement (Thermo Fisher Scientific). The culture supernatants were screened using direct enzyme-linked immunosorbent assay (ELISA) for the binding to synthetic peptide and recombinant protein of hTERT purified from E. coli. The Animal Care and Use Committee of Tohoku University approved the animal experiments about hybridoma production in this study.

2.6. Verification of the Specificity of Anti-hTERT Antibodies Using ELISA. Synthetic peptide and recombinant protein of hTERT were immobilized on Nunc Maxisorp 96-well microplates (Thermo Fisher Scientific) at a concentration of $1 \mu \mathrm{g} / \mathrm{ml}$ and $5 \mu \mathrm{g} / \mathrm{ml}$, respectively, for $30 \mathrm{~min}$. After blocking with $1 \%$ bovine serum albumin (BSA) containing $0.05 \%$ Tween 20 in PBS, the plates were incubated with culture supernatant followed by 1:3000 diluted peroxidaseconjugated anti-mouse IgG (Dako, Glostrup, Denmark). The enzymatic reaction was conducted with a 1-Step Ultra TMB-ELISA (Thermo Fisher Scientific). The optical density was measured at $655 \mathrm{~nm}$ using an iMark microplate reader (Bio-Rad Laboratories, Berkeley, CA).

2.7. Immunoprecipitation-RNA-Dependent RNA Polymerase (IP-RdRP) Assay. $1 \times 10^{7}$ cells treated with nocodazole were lysed in $1 \mathrm{ml}$ of lysis buffer A [0.5\% NP-40, $20 \mathrm{mM}$ Tris- $\mathrm{HCl}$ (pH 7.4) and $150 \mathrm{mM} \mathrm{NaCl}$. After sonication, cell lysates were cleared of insoluble material by centrifugation at $21,000 \times \mathrm{g}$ at $4^{\circ} \mathrm{C}$ for $15 \mathrm{~min}$. One milliliter of the lysates was preabsorbed 2 times with $40 \mu \mathrm{L}$ of Protein L Agarose (Sigma-Aldrich) for $30 \mathrm{~min}$ at $4^{\circ} \mathrm{C}$. The preabsorbed lysates were mixed with $10 \mu \mathrm{g}$ of an anti-human TERT monoclonal antibody and $40 \mu \mathrm{L}$ of Protein L Agarose and incubated overnight at $4^{\circ} \mathrm{C}$. The remaining of the IP-RdRP assay was performed as described previously [28, 29].

2.8. Immunohistochemistry for TERT. All sections were deparaffinized in xylene and rehydrated in an ethanol gradient. Sections were subjected to antigen retrieval by microwaving in $10 \mathrm{mM}$ citrate buffer ( $\mathrm{pH} 6.0$ ) for $20 \mathrm{~min}\left(95^{\circ} \mathrm{C}, 500 \mathrm{~W}\right)$. Endogenous peroxidase activity was quenched with $0.3 \%$ $\mathrm{H}_{2} \mathrm{O}_{2}$ in methanol. The sections were then incubated with a primary antibody [TMab-6; mouse IgM, kappa; diluted in $1: 2000(0.5 \mu \mathrm{g} / \mathrm{mL})]$ at room temperature for $90 \mathrm{~min}$. The optimal concentration of the primary antibody was determined by a serial dilution of the antibody. After rinsing, the sections were subjected to the polymer-immunocomplex method using EnvisionTM (Dako) for enhancing. 3,3' Diaminobenzidine tetrahydrochloride (DAB; Dojindo, Kumamoto, Japan) was used as the chromogen and hematoxylin as the counterstain. To confirm the specificity of the TERT expression state in glioma cells, negative control reaction was performed by omission of the primary antibodies, and preabsorption test of the antibodies was also conducted using the synthetic peptide of hTERT (Sigma-Aldrich) at a final concentration of $0.1 \mathrm{mg} / \mathrm{mL}$. The percentage of TERTpositive cells in each case was semiquantitatively evaluated as $1+$ (a few weakly positive cells), $2+$ (positive cells $<50 \%$ ), and $3+$ (positive cells $\geq 50 \%$ ). We also immunostained the glioma samples with the most widely used, commercially available antibody for TERT (sc-7215; Santa Cruz Biotechnology, Santa Cruz, CA), but the staining was rather faint than that by TMab-6 (Supplementary Figure 1). Thus, we evaluated immunohistochemical observations of TERT in all the glioma samples with our newly developed TMab-6.

2.9. Western Blotting. Immunoblotting was performed according to a modified version of the previously reported methods [30, 31]. Snap-frozen tissue samples were lysed and homogenized with radioimmunoprecipitation assay (RIPA) lysis buffer [ $50 \mathrm{mM}$ Tris- $\mathrm{HCl}, 150 \mathrm{mM} \mathrm{NaCl}, 1 \% \mathrm{NP}-40$, $0.5 \%$ sodium deoxycholate, and $0.1 \%$ sodium dodecyl sulfate 
TABLE 1: Molecular information of the cases.

\begin{tabular}{|c|c|c|c|c|c|c|c|c|}
\hline \multirow{2}{*}{ Classification } & \multirow{2}{*}{ Number } & \multicolumn{2}{|c|}{ TERT $\mathrm{mt}$} & \multirow{2}{*}{$\begin{array}{l}\text { TERT SNP } \\
\text { T349C (\%) }\end{array}$} & \multirow{2}{*}{$\begin{array}{c}\text { IDH1/2 mt } \\
(\%) \\
\end{array}$} & \multirow{2}{*}{$\begin{array}{c}\text { p53 IHC } \\
(\%)\end{array}$} & \multirow{2}{*}{$\begin{array}{c}\text { ATRX IHC } \\
(\%) \\
\end{array}$} & \multirow{2}{*}{$\begin{array}{c}1 \mathrm{p} / 19 \mathrm{q}-\text { codel } \\
(\%)\end{array}$} \\
\hline & & $\mathrm{C} 228 \mathrm{~T}(\%)$ & $\mathrm{C} 250 \mathrm{~T}(\%)$ & & & & & \\
\hline Astrocytic with IDH-mutant (DA/AA) & 11 & 0 & 0 & 54.5 & 100 & 72.7 & 0 & 0 \\
\hline Oligodendroglial & 10 & 70 & 30 & 30 & 100 & 0 & 100 & 100 \\
\hline GBM & 11 & 45.5 & 18.2 & 90.9 & 0 & 27.3 & 100 & 0 \\
\hline DA/AA with IDH-wildtype & 9 & 33.3 & 11.1 & 33.3 & 0 & 0 & 100 & 0 \\
\hline Total & 41 & 36.6 & 14.6 & 53.7 & 51.2 & 26.8 & 73.2 & 24.4 \\
\hline
\end{tabular}

AA, anaplastic astrocytoma; codel, codeletion; DA, diffuse astrocytoma; GBM, IDH-wildtype glioblastoma; IHC, immunohistochemistry; mt, mutation.

(SDS)] from Boston BioProducts (Boston, MA). Protein concentration of each sample was determined using the BCA kit (Thermo Fisher Scientific) according to the manufacturer's instructions. Equal amounts of protein extracts were separated by electrophoresis on Any $\mathrm{kD}$ Mini-PROTEAN TGX Precast Gels (Bio-Rad) and then transferred to a nitrocellulose membrane with Trans-Blot Turbo Transfer System (Bio-Rad). The membrane was probed with the primary antibodies against TERT (sc-7215) and glyceraldehyde phosphate dehydrogenase (GAPDH) (D16H11; Cell Signaling Technology, Danvers, MA), followed by secondary antibodies conjugated to horseradish peroxidase. TMab-6 was not available for the immunoblotting study because TMab6 was developed specifically for the immunohistochemical purpose and produced nonspecific bands on Western blots. The immunoreactivity was detected with Super Signal West Pico Chemiluminescent Substrate or West Femto Trial kit (Thermo Fisher Scientific). Quantitative densitometry analysis was performed with image analysis software (ImageJ version $1.49, \mathrm{NIH}$ ).

2.10. Quantitative Reverse Transcription Polymerase Chain Reaction ( $q R T-P C R)$ Analysis. Total RNA was extracted by the use of RNeasy Plus Mini Kit (QIAGEN). Firststrand cDNA was synthesized by the use of iScript ${ }^{\mathrm{TM}}$ RT Supermix for RT-qPCR (Bio-Rad). Real-time RT-PCR was performed with the $\mathrm{SYBR}^{\circledR}$ Premix Ex $\mathrm{Taq}^{\mathrm{TM}}$ II (Tli RNaseH Plus) (Takara, Kyoto, Japan) on Thermal Cycler Dice Real Time System TP800 (Takara) following the manufacturer's instructions. $\beta$-Actin was used as an endogenous control. Primer sequences were provided in Supplementary Table 2.

2.11. Statistical Analysis. The data were routinely compared between three or more groups with a two-way analysis of variance (ANOVA) followed by post hoc test Bonferroni corrections for TERT expression using the Statistical Package for the Social Sciences software package (Version 16.0; SPSS, Chicago, IL, USA). $p$ value below 0.05 was considered statistically significant.

\section{Results}

3.1. Molecular Profiling of the Cases. All 41 tumor samples and 4 control brain samples were subjected to histological as well as molecular genetic diagnostics, and mutations at C228 and C250 of the TERT promoter region were subsequently examined for every case by Sanger sequencing, successfully detecting these two reported hotspot mutations (Figure 1(b)). As a result, mutations in the TERT promoter were found in 21 out of 41 tumor samples (51.2\%). Consistent with previous reports [5], oligodendroglial tumors (10 mutations out of 10 tumors: $100 \%$ ) and IDH-wildtype GBM (7 mutations out of 11 tumors: $63.6 \%$ ) possessed high incidence of TERT promoter mutations whereas no astrocytic tumors with $I D H$ mutations showed them (Figure 1(c)). Interestingly, some of the $I D H$-wildtype diffuse astrocytomas demonstrated hotspot mutations in the TERT promoter ( 4 mutations out of 9 tumors: $44.4 \%$ ). In addition to the TERT promoter hotspot mutations, we identified a common single nucleotide polymorphism (SNP) (T349C) [32] across all types of gliomas we investigated (Figure 1(d)): IDH-mutant astrocytic tumors (6 out of the 11 tumors: 54.5\%), oligodendroglial tumors (3 out of the 10 tumors: $30.0 \%$ ), GBM (10 out of the 11 tumors: $90.9 \%)$, and IDH-wildtype diffuse astrocytomas (3 out of the 9 tumors: $33.3 \%$ ). The molecular profile of the cases was summarized in Table 1 and Supplementary Table 1.

3.2. Production and Validation of a Novel Anti-TERT Monoclonal Antibody Available for Human Tissue. In order to evaluate the expression of TERT protein in human glioma tissue, we developed and screened novel anti-hTERT monoclonal antibodies that will be applicable to human tissue sections. We generated about 10,000 hybridomas from two mice and picked up about 150 wells by using ELISA. Next, we screened by using ELISA (synthetic peptide and recombinant protein), western blotting (recombinant protein and GBM cell line lysate), and immunohistochemistry. Some clones were excluded because of nonspecific reactivity by these analyses. Finally, we established nine hybridomas as TMab clones. Among these, TMab-6 showed sensitive reactivity in immunohistochemistry with human tumor tissue of anaplastic oligodendroglioma (IDH-mutant and 1p/19q-codeleted), glioblastoma (IDH-wildtype), and anaplastic astrocytoma (IDH-mutant). In contrast, the other clones did not show sensitive and specific reactivity in immunohistochemistry. We used clone TMab- 6 for further studies. Next, we validated the specificity of TMab- 6 by immunoprecipitation-based assessment of the enzymatic activity of TERT as well as antibody preabsorption test for tissue sections (Figures 2(a) and 2(b)). Since TMab-6 was IgM clone, we immunoprecipitated endogenous TERT complex by protein L beads from HeLa cells synchronized in mitotic phase by nocodazole [28, 29]. We found that a novel anti-hTERT mAb TMab-6 specifically 


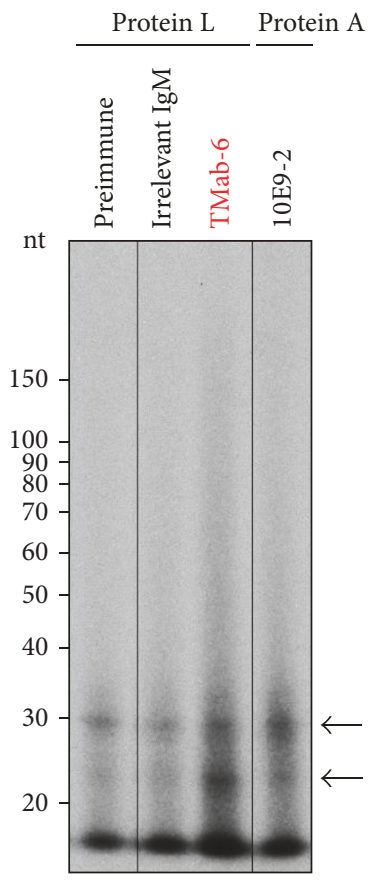

(a)

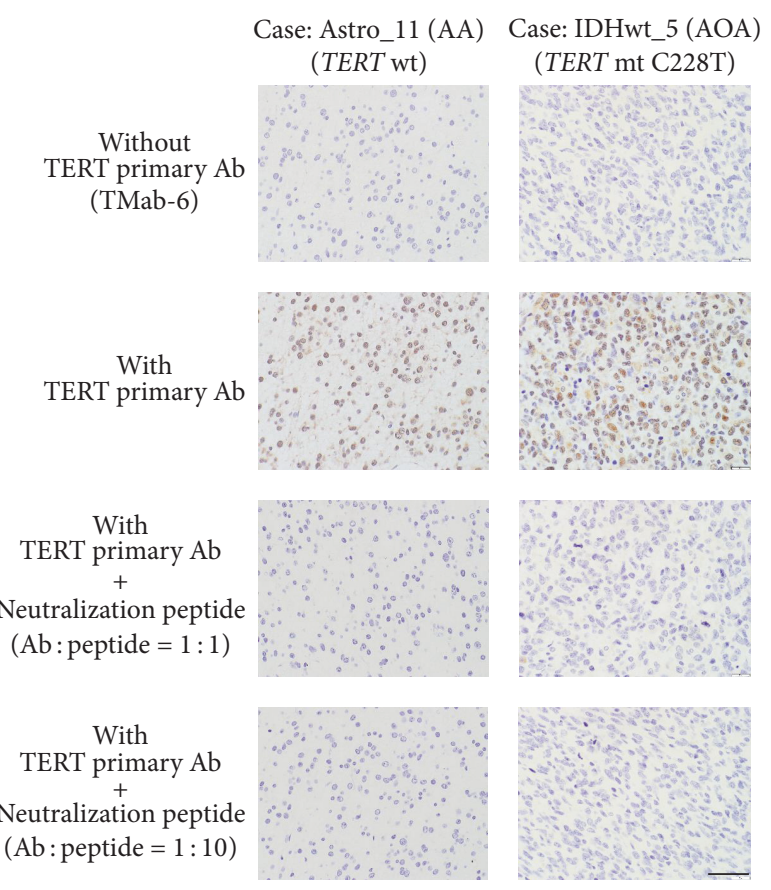

(b)

U87 malignant glioma cell line [TERT mt (C228T)]

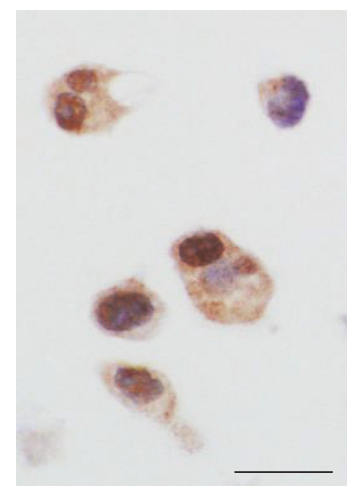

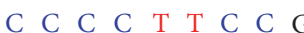

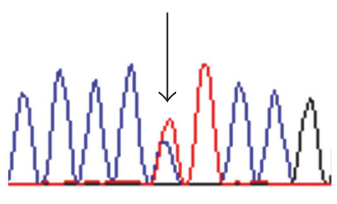

TERT mutation

(C228T)

(c)

FIGURE 2: Validation of a newly developed TERT-specific antibody (TMab-6) usable for human glioma tissue. (a) Endogenous TERT was immunoprecipitated with an anti-human TERT mAb (TMab-6) followed by an RNA-dependent RNA polymerase (RdRP) assay using HeLa cells treated with nocodazole. Arrows indicate RdRP products. The preimmune (without antibodies) and irrelevant IgM lanes are a negative control, and the 10E9-2 antibody (MBL, Nagoya, Japan) lane is a positive control. nt, nucleotide. (b) The IDH-mutant astrocytoma (anaplastic astrocytoma: AA) and IDH-wildtype diffuse astrocytoma (Histologically anaplastic oligoastrocytoma: AOA) cases were analyzed for TERT protein expression. TMab-6 specifically recognized the nuclei of the tumor cells, and immunoreactivity was not detected in the negative control section without the application of TMab-6. An antibody absorption test with synthetic neutralization peptide of TERT further validated the specificity of TMab-6 as an anti-TERT antibody which is applicable to human glioma tissue. (c) TMab-6 Immunostaining of U87 malignant glioma cell lines with a TERT hotspot mutation (C228T) showed strong nuclear immunoreactivity for TERT. Ab, antibody; $\mathrm{mt}$, mutation. Scale bar $=40 \mu \mathrm{m}$.

recognized human TERT protein, and that it could be applied for immunohistochemistry on the human glioma tissue sections. We further confirmed the nuclear staining of TERT by TMab-6 in U87 malignant glioma cell lines (Figure 2(c)) that were previously reported to possess TERT hotspot mutations as well as upregulated TERT transcripts $[5,33]$.
3.3. Expression of TERT in Human Glioma Tissue. After confirming the specificity of a newly developed anti-TERT monoclonal antibody TMab-6, we performed immunohistochemical staining on sections of nonneoplastic adult human cerebral tissues and all glioma samples including $I D H$-mutated astrocytoma, oligodendroglioma, IDH-wildtype GBM, and $I D H$-wildtype diffuse astrocytoma and examined the staining 
$\mathrm{H} \& \mathrm{E}$
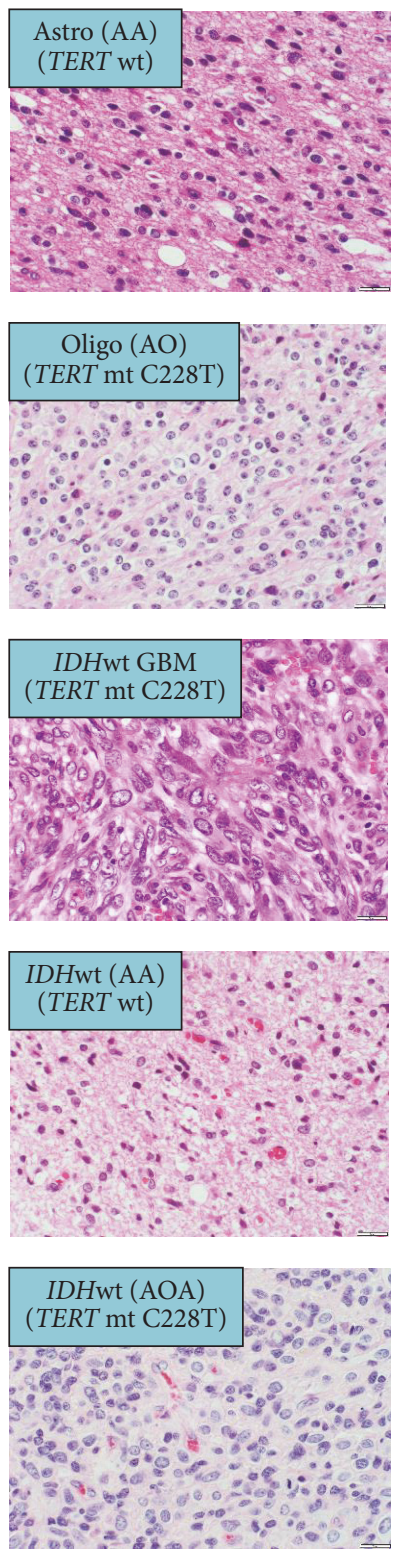

TERT
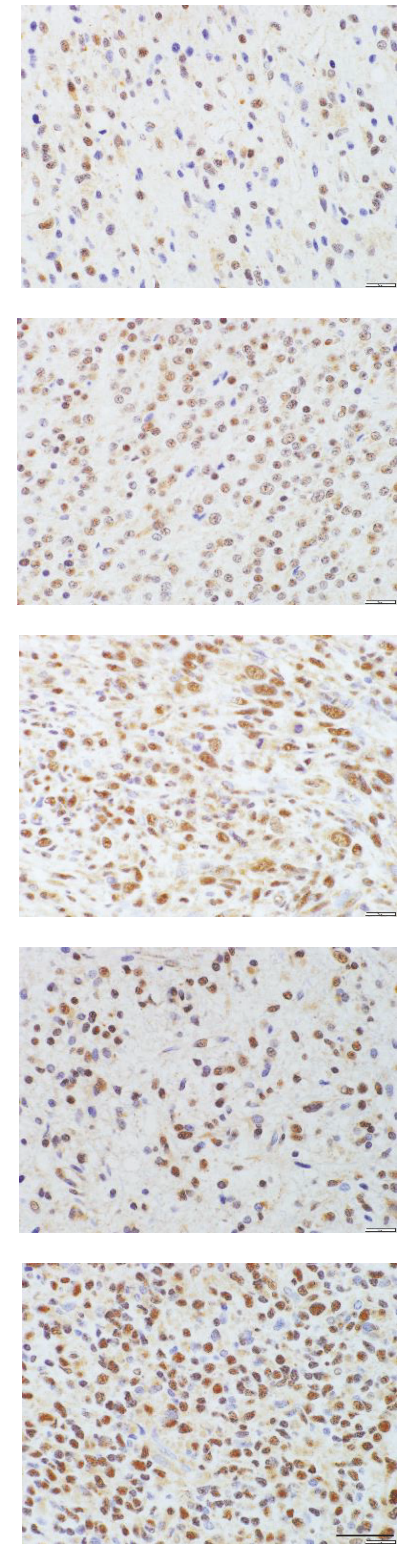

(a)
Control (nonneoplastic tissue)

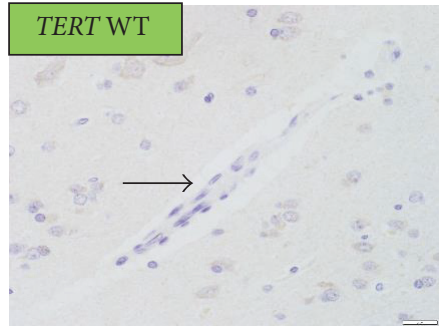

Case: Astro_4 (AA)

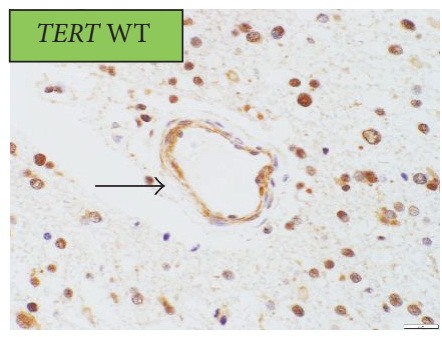

Case: Oligo_10 (AO)

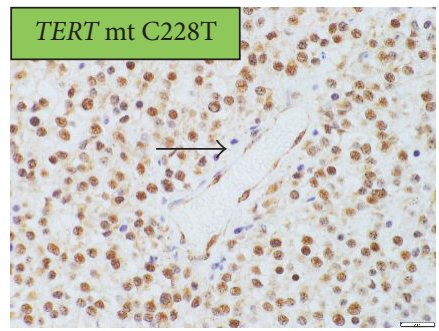

Case: GBM_5 (IDHwt GBM)

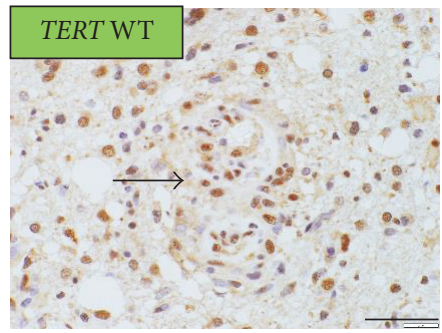

(b)

FIGURE 3: TERT expression in human glioma samples. (a) TMab-6 immunostaining of the glioma samples demonstrated strong nuclear as well as some cytoplasmic staining in tumor cells. Representative tumors were shown in the astrocytic, oligodendroglial, IDH-wildtype GBM, and $I D H$-wildtype diffuse astrocytoma groups. Glioma cells in all the cases showed nuclear immunostaining of TERT to a various degree. (b) TMab-6 immunostaining of vascular endothelial cells (arrows) in the nonneoplastic cerebral tissues, TERT-wildtype AA, TERT-mutated $\mathrm{AO}$, and TERT-mutated GBM. AA, anaplastic astrocytoma; AO, anaplastic oligodendroglioma; AOA, anaplastic oligoastrocytoma; GBM, $I D H$-wildtype glioblastoma; mt, mutation; wt/WT, wildtype. Scale bar $=40 \mu \mathrm{m}$.

patterns of TERT protein in them. Immunohistochemical observations using TMab-6 demonstrated strong staining in the nucleus and some cytoplasmic staining of the glioma cells (Figure 3(a)), and TERT immunoreactivity was not observed in the normal tissue adjacent to the tumor tissue. Surprisingly, glioma cells in all the cases showed nuclear immunostaining for TERT to various degrees compared with the cells in nonneoplastic tissues (Figure 3(a)) although some intratumoral heterogeneity of the nuclear staining was observed. Some reactive astrocytes in cerebral parenchyma from the cases with hippocampal sclerosis and cortical dysplasia showed nonspecific, weak cytoplasmic staining (Supplementary Figure 2). Interestingly, endothelial cells of the tumor vessels also displayed nuclear immunostaining in both TERT-mutated and wildtype tumors, whereas vascular endothelial cells in the nonneoplastic tissues did not show any specific TERT immunoreactivity in their nuclei (Figure 3(b)). 
3.4. Semiquantitative Evaluation of TERT Expression Associated with TERT Promoter Mutations in Human Gliomas. Having confirmed the findings that all the glioma cells we analyzed showed nuclear immunostaining of TERT to various degrees, we further examined the association of the TERT promoter mutations with TERT immunoreactivity in our glioma samples. Unexpectedly, immunohistochemistry using TMab-6 demonstrated that immunoreactivity for TERT did not correspond to the mutational status of the TERT promoter region across glioma samples (Figure 4(a)). Although the staining was weak, nuclear immunoreactivity for TERT was also confirmed in both TERT-wildtype and mutated gliomas with the commercially available antibody for TERT (sc-7215) (Supplementary Figure 1). In addition to the hotspot mutations in the TERT promoter (C228T and C250T), the presence of a common SNP in the TERT promoter (T349C) was not associated with TERT immunoreactivity (Supplementary Table 1). TERT immunohistochemistry was not capable of identifying the differences between TERT-mutated gliomas and TERT-nonmutant gliomas (Figure $4(\mathrm{a}))$. The scores might be relevant to tumor grading and aggressiveness represented by $I D H$-wildtype GBMs and $I D H$-wildtype diffuse astrocytomas (Figure 4(a)), both of which behave aggressively with a dismal prognosis $[1,4,14$, 21]. Finally, the relationship between the TERT promoter mutations, the expression levels of TERT protein, and tumor entity was further verified by Western blotting (Figure 4(b)), and the level of TERT mRNA was not parallel with its mutational status across all the glioma samples (data not shown).

\section{Discussion}

The mechanism of TERT upregulation in cancer has largely remained unknown to date. Recurrent mutations at two hotspot regions (C228T and C250T) in the TERT promoter have been reported in various types of cancers $[2,3,5]$, and a recent report outlined the mechanism that TERT promoter mutations reactivate TERT expression [34]. In diffuse gliomas, the sole mechanism to upregulate TERT mRNA is considered to be two hotspot mutations in the TERT promoter region. However, the level of TERT transcripts varies widely in expression among tumors including gliomas [5, 7], skin cancers [2, 3, 35], thyroid cancers [7], hepatocellular carcinomas [36], bladder cancers [37], and malignant lymphomas [38]. The levels of TERT mRNA tend to be increased in TERT-mutated tumors in comparison with TERT-wildtype tumors, but it is not necessarily the case for the individual tumors, indicating that mutations in the TERT promoter alone could not explain the wide variety in the expression of TERT transcripts. Further, to the best of our knowledge, only one report has so far investigated the expression of TERT protein in gliomas, and any difference was not detected between TERT-mutated and TERT-wildtype GBMs [7].

In the present study, we compared the expression of TERT protein with its mutational status in adult gliomas. In line with the previous studies, TERT expression was confirmed in the nuclei of oligodendroglial tumors and $I D H$-wildtype GBM which possess either of two hotspot mutations in the promoter regions of TERT. Surprisingly, however, even $I D H$ mutant astrocytic tumors (DA/AA) without known hotspot mutations in the TERT promoter displayed certain amount of TERT immunoreactivity in their nuclei. Additionally, qRTPCR study did not demonstrate any significant correlation between TERT promoter mutations and the level of TERT mRNA expression. Considering our findings that immunoprecipitation with TMab- 6 did demonstrate the same blotting bands as the commercially available antibody (10E9-2/sc7215) and TMab- 6 and sc-7215 showed the similar immunostaining pattern in the tumor, TERT immunoreactivity is surely increased in tumor tissue in comparison with normal tissue although the possibility of any unknown crossreactivity cannot be completely ruled out. The finding that the TERT immunoreactivity in endothelial as well as tumor cells is strictly limited to the neoplastic tissue further supports the idea that TMab- 6 detects TERT protein closely bounded to the tumor environment composed of neoplastic tissue and neovasculatures.

Our findings of an unexpected increase in the expression of TERT protein even in the TERT-wildtype gliomas raise the possibility that the expression of TERT mRNA and protein could be regulated by the mechanism other than its promoter mutations, including via epigenetic factors. In addition to reported hotspot mutations, certain SNPs in the TERT promoter region (T349C) were reported to suppress TERT transcripts [32], but we could not confirm any association of SNPs with TERT protein expression, further supporting our hypothesis that TERT expression could be epigenetically regulated.

There could be several mechanisms to increase the expression of TERT protein in TERT-wildtype tumors. It has been reported that DNA hypermethylation of the TERT promoter is associated with TERT upregulation in pediatric brain tumors [8] and other tumors [39], and histone acetylation at the TERT promoter regions may also be relevant to its expression [9]. IDH-mutant diffuse astrocytic tumors often demonstrate the glioma-CpG island methylator phenotype (G-CIMP) [40, 41], and the nuclear TERT staining in diffuse astrocytomas might be epigenetically caused by hypermethylation of the TERT promoter [10]. Additionally, a number of human tumors maintain their telomeres by a telomeraseindependent mechanism termed alternative lengthening of telomeres (ALT) caused by such abnormalities as loss of ATRX [42, 43], and this might somehow affect the expression level of TERT.

In addition to its applicability to the molecular diagnostics of gliomas, TERT could be a good therapeutic target against diffuse gliomas which are a potentially malignant, incurable brain tumor in human. A novel TERT-targeting therapy $[44,45]$ would be expected to specifically target IDH-wildtype GBM including primary brain tumor initiating cells and 1p/19q-codeleted oligodendroglioma which have characteristically possessed hotspot mutations in the TERT promoter region $[5,33]$. Our unexpected findings suggest that a specific TERT-targeting therapy might be a promising therapeutic strategy against all types of gliomas by directly targeting tumor cells with an indirect antiangiogenic effect on tumor vasculature like the antiangiogenic agent 

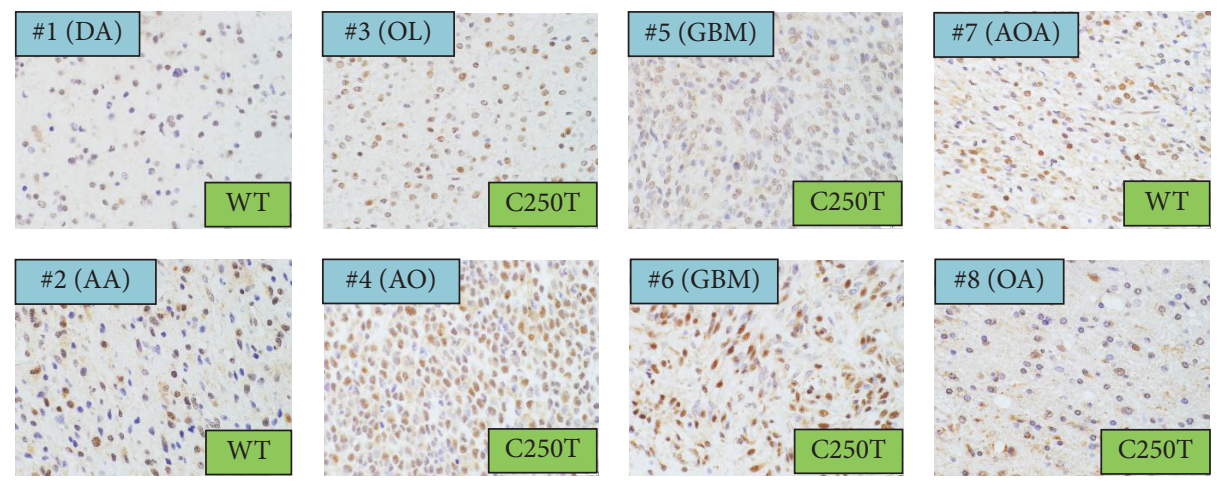

TERT immunoreactivity
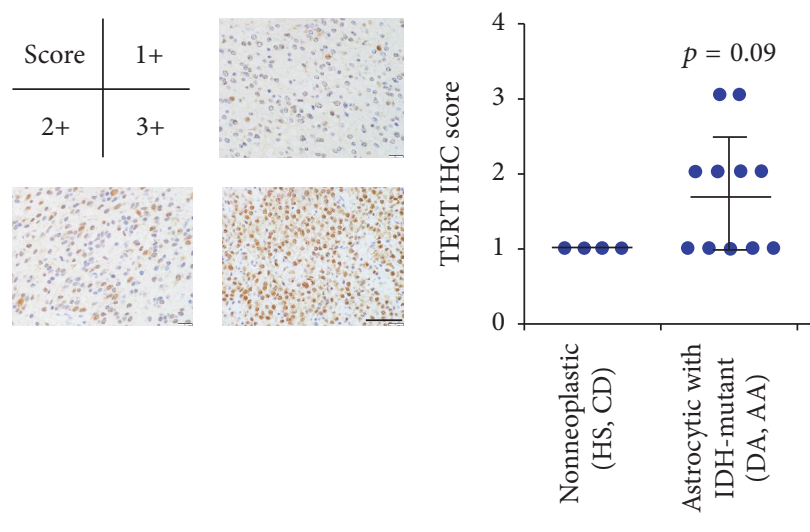

(a)

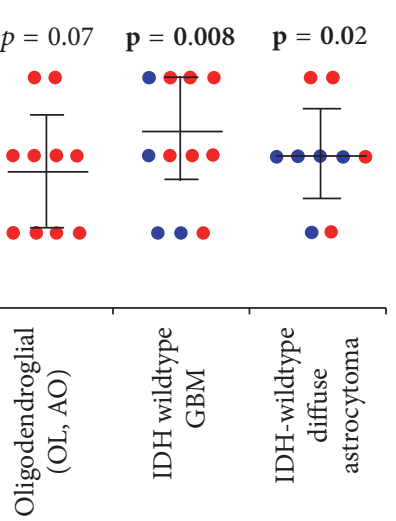

TERT protein expression
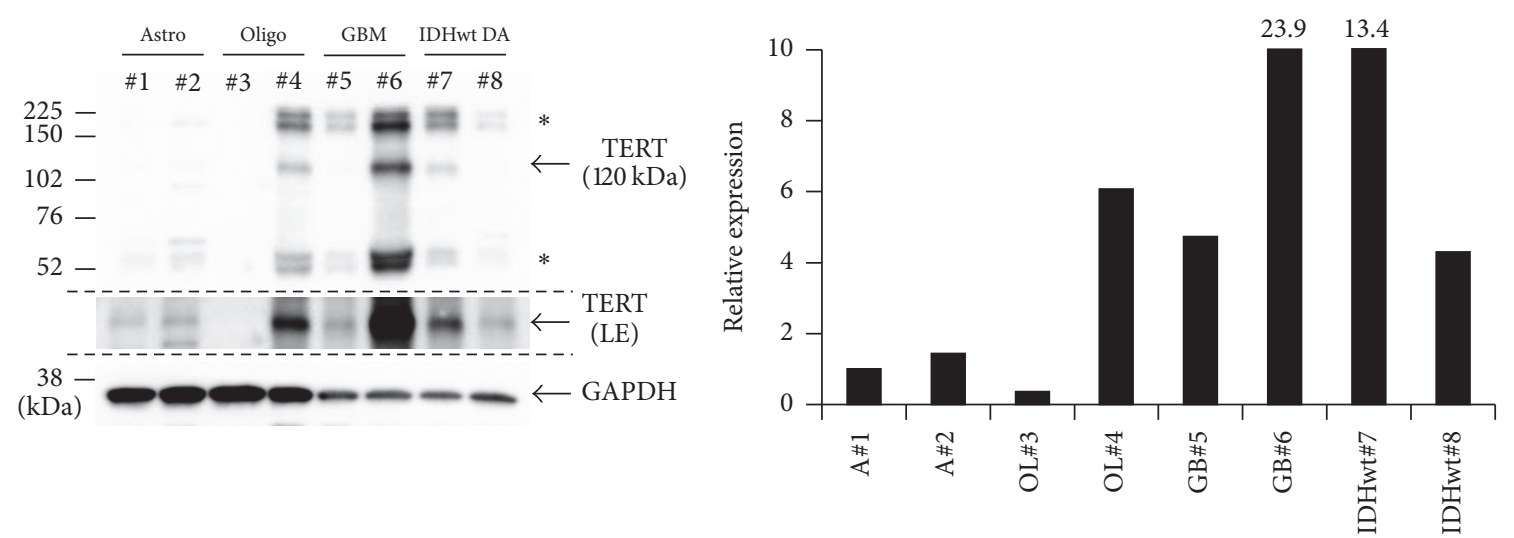

(b)

FIgURE 4: Association of TERT promoter mutations and its immunoreactivity in human gliomas. (a) Immunostaining of TERT by TMab-6 demonstrated that TERT immunoreactivity did not correspond to the mutational status of the TERT promoter region across glioma samples. Semiquantitative analysis of the TERT-immunostaining, scoring the staining intensities as $1+$ (a few weakly positive cells), $2+$ (positive cells $<$ $50 \%$ ), and $3+$ (positive cells $\geq 50 \%$ ). Blue dots represent TERT-wildtype cases and red denotes TERT-mutated cases. $P$ values for nonneoplastic tissue versus each glioma entity. (b) An analysis on the relationship of the TERT promoter mutations, the expression level of TERT protein and each tumor entity by Western blotting with the most widely used, commercially available TERT antibody (sc-7215). A bar graph showed the quantification of TERT expression in each case, normalized by a loading control GAPDH expression. ${ }^{*}$ Nonspecific bands. LE, long exposure. AA, anaplastic astrocytoma; AO, anaplastic oligodendroglioma; AOA, anaplastic oligoastrocytoma; CD, cortical dysplasia; DA, diffuse astrocytoma; GBM, IDH-wildtype glioblastoma; HS, hippocampal sclerosis; OA, oligoastrocytoma; OL, oligodendroglioma; IDHwt, $I D H$-wildtype diffuse astrocytoma; wt/WT, wildtype. Scale bar $=40 \mu \mathrm{m}$.

bevacizumab, a humanized monoclonal antibody against vascular endothelial growth factor (VEGF) ligands [46], but the additional studies are essential to demonstrate that the presence of TERT protein actually implies more aggressive biology for the recommendation of treatment based on TERT immunohistochemistry.

The limitation of our study is that we have not directly measured the telomerase activity itself. Indeed, some reports 
demonstrated that TERT expression and its biological activity are not necessarily parallel [12]. Further, there could be the possibility that this novel antibody is picking up baseline TERT protein that may not be associated with increased telomerase efficiency or activity. It would be thus important to further validate/specify the antibody and analyze the relationship between histological TERT immunoreactivity and its enzymatic activity as a next step. Future studies are also necessary to unravel the contributing factors in upregulating the protein expression of TERT to examine if TERT protein expression could be the biomarker for the applicability of TERT-targeting therapeutics in the future.

Together, we developed a novel TERT-specific antibody, and it is sensitively and specifically applicable to immunohistochemistry for TERT in human glioma tissue. Immunohistochemistry with this TERT-specific antibody could not play the diagnostic surrogate for TERT promoter mutations, but we could find an unexpected increase in TERT immunoreactivity across all types of gliomas and tumor vasculature. This study is the first extensive analysis on the expression of TERT protein in human glioma tissue and suggests that TERT expression could be regulated by various mechanisms including epigenetic modifications. Future studies with the measurement of TERT activity could be useful for facilitating our understanding of telomere biology in cancer including gliomas.

\section{Disclosure}

A part of this work was presented at 11th European Congress of Neuropathology (July 6-9, 2016, Bordeaux, France) and 13th Asian Society for Neuro-Oncology (September 11-14, 2016, Sydney, Australia).

\section{Conflicts of Interest}

The authors declare no conflicts of interest.

\section{Authors' Contributions}

K. Masui and T. Komori contributed equally to the work.

\section{Acknowledgments}

The authors would like to thank Mr. Mizuho Karita, Mr. Takashi Sakayori (Tokyo Women's Medical University), and Dr. Mami Yasukawa (Division of Cancer Stem Cell, National Cancer Center Research Institute) for their excellent technical support. This research was supported in part by AMED under Grant nos. JP17am0301010 (Yukinari Kato), JP17am0101078 (Yukinari Kato), and JP17ae0101028 (Yukinari Kato), by JSPS KAKENHI Grant no. $17 \mathrm{~K} 07299$ (Mika K. Kaneko) and Grant no. 16K10748 (Yukinari Kato), and by the Cell Science Research Foundation, Grant-inAid from the Tokyo Biochemical Research Foundation, and JSPS KAKENHI Grant nos. 15K19067 and 17K15672 (Kenta Masui).

\section{Supplementary Materials}

Supplementary Table 1. Detailed information of the cases.

Supplementary Table 2. Primer information.

Supplementary Figure 1. Immunostaining of TERT protein in human gliomas with TMab-6 and sc-7215. We immunostained TERT-wildtype and mutant gliomas with our newly developed TMab- 6 and the most widely used, commercially available sc-7215. Both antibodies detected nuclear immunoreactivity of TERT in TERT-wildtype and mutated gliomas although the staining intensities were variable. AA, anaplastic astrocytoma; AO, anaplastic oligodendroglioma. Scale bar $=40 \mu \mathrm{m}$.

Supplementary Figure 2. Immunostaining of TERT protein in human nonneoplastic brain tissue. We immunostained the cases of TERT-wildtype hippocampal sclerosis and cortical dysplasia with our newly developed TMab-6 $(0.5 \mu \mathrm{g} / \mathrm{mL})$. Some reactive astrocytes showed nonspecific, weak cytoplasmic staining although any nuclear staining of TERT was not observed in them.

\section{References}

[1] D. J. Brat, R. G. Verhaak, K. D. Aldape et al. et al., "Comprehensive, integrative genomic analysis of diffuse lower-grade gliomas," The New England Journal of Medicine, vol. 372, pp. 2481-2498, 2015.

[2] S. Horn, A. Figl, P. S. Rachakonda et al., "TERT promoter mutations in familial and sporadic melanoma," Science, vol. 339, no. 6122, pp. 959-961, 2013.

[3] F. W. Huang, E. Hodis, M. J. Xu, G. V. Kryukov, L. Chin, and L. A. Garraway, "Highly recurrent TERT promoter mutations in human melanoma," Science, vol. 339, no. 6122, pp. 957-959, 2013.

[4] J. E. Eckel-Passow, D. H. Lachance, A. M. Molinaro et al. et al., "Glioma Groups Based on 1p/19q, IDH, and TERT Promoter Mutations in Tumors," The New England Journal of Medicine, vol. 372, pp. 2499-2508, 2015.

[5] H. Arita, Y. Narita, S. Fukushima et al., "Upregulating mutations in the TERT promoter commonly occur in adult malignant gliomas and are strongly associated with total 1p19q loss," Acta Neuropathologica, vol. 126, no. 2, pp. 267-276, 2013.

[6] H. Arita, Y. Narita, H. Takami et al., "TERT promoter mutations rather than methylation are the main mechanism for TERT upregulation in adult gliomas," Acta Neuropathologica, vol. 126, no. 6, pp. 939-941, 2013.

[7] J. Vinagre, A. Almeida, H. Pópulo et al., "Frequency of TERT promoter mutations in human cancers," Nature Communications, vol. 4, article 2185, 2013.

[8] P. Castelo-Branco, S. Choufani, S. Mack et al., "Methylation of the TERT promoter and risk stratification of childhood brain tumours: An integrative genomic and molecular study," The Lancet Oncology, vol. 14, no. 6, pp. 534-542, 2013.

[9] B. Zhang, J. Chen, A. S. L. Cheng, and B. C. B. Ko, "Depletion of sirtuin 1 (SIRT1) leads to epigenetic modifications of telomerase (TERT) gene in hepatocellular carcinoma cells," PLoS ONE, vol. 9, no. 1, Article ID e84931, 2014. 
[10] S. Ohba, J. Mukherjee, T.-C. Johannessen et al., "Mutant IDH1 expression drives TERT promoter reactivation as part of the cellular transformation process," Cancer Research, vol. 76, no. 22, pp. 6680-6689, 2016.

[11] N. W. Kim, M. A. Piatyszek, K. R. Prowse et al., "Specific association of human telomerase activity with immortal cells and cancer," Science, vol. 266, no. 5193, pp. 2011-2015, 1994.

[12] M. Ceccarelli, F. P. Barthel, T. M. Malta et al., "Molecular profiling reveals biologically discrete subsets and pathways of progression in diffuse glioma," Cell, vol. 164, pp. 550-563, 2016.

[13] T. Komori, "Updated 2016 WHO classification of tumors of the CNS: turning the corner where molecule meets pathology," Brain Tumor Pathology, vol. 34, no. 4, pp. 139-140, 2017.

[14] D. N. Louis, A. Perry, G. Reifenberger et al., “The 2016 World Health Organization Classification of Tumors of the Central Nervous System: a summary," Acta Neuropathologica, vol. 131, no. 6, pp. 803-820, 2016.

[15] A. Perry, "WHO's arrived in 2016! An updated weather forecast for integrated brain tumor diagnosis," Brain Tumor Pathology, vol. 33, no. 3, pp. 157-160, 2016.

[16] D. N. Louis, A. Perry, P. Burger et al., "International society of neuropathology-haarlem consensus guidelines for nervous system tumor classification and grading," Brain Pathology, vol. 24, no. 5, pp. 429-435, 2014.

[17] K. Masui, P. S. Mischel, and G. Reifenberger, "Molecular classification of gliomas," Handbook of Clinical Neurology, vol. 134, pp. 97-120, 2016.

[18] H. Takami, A. Yoshida, S. Fukushima et al., "Revisiting TP53 mutations and immunohistochemistry - A comparative study in 157 diffuse gliomas," Brain Pathology, vol. 25, no. 3, pp. 256265,2015

[19] X.-Y. Liu, N. Gerges, A. Korshunov et al., "Frequent ATRX mutations and loss of expression in adult diffuse astrocytic tumors carrying IDH1/IDH2 and TP53 mutations," Acta Neuropathologica, vol. 124, no. 5, pp. 615-625, 2012.

[20] D. E. Reuss, F. Sahm, D. Schrimpf et al., "ATRX and IDH1$\mathrm{R} 132 \mathrm{H}$ immunohistochemistry with subsequent copy number analysis and IDH sequencing as a basis for an "integrated" diagnostic approach for adult astrocytoma, oligodendroglioma and glioblastoma," Acta Neuropathologica, vol. 129, no. 1, pp. 133-146, 2015.

[21] K. Aldape, G. Zadeh, S. Mansouri, G. Reifenberger, and A. von Deimling, "Glioblastoma: pathology, molecular mechanisms and markers," Acta Neuropathologica, vol. 129, no. 6, pp. 829848, 2015.

[22] S. Ogasawara, Y. Fujii, M. K. Kaneko et al., "Establishment of Anti-Human ATRX Monoclonal Antibody AMab-6," Monoclonal Antibodies in Immunodiagnosis and Immunotherapy, vol. 35, no. 5, pp. 254-258, 2016.

[23] M. J. Van Den Bent, A. F. Carpentier, A. A. Brandes et al., "Adjuvant procarbazine, lomustine, and vincristine improves progression-free survival but not overall survival in newly diagnosed anaplastic oligodendrogliomas and oligoastrocytomas: A randomized European Organisation for Research and Treatment of Cancer phase III trial," Journal of Clinical Oncology, vol. 24, no. 18, pp. 2715-2722, 2006.

[24] H. Arita, Y. Narita, Y. Matsushita et al., "Development of a robust and sensitive pyrosequencing assay for the detection of IDH1/2 mutations in gliomas," Brain Tumor Pathology, vol. 32, no. 1, pp. 22-30, 2014.
[25] H. Arita, Y. Narita, A. Yoshida, N. Hashimoto, T. Yoshimine, and K. Ichimura, "IDH1/2 mutation detection in gliomas," Brain Tumor Pathology, vol. 32, no. 2, pp. 79-89, 2015.

[26] Y. Fujii, M. K. Kaneko, and Y. Kato, "MAP Tag: A Novel Tagging System for Protein Purification and Detection," Monoclonal Antibodies in Immunodiagnosis and Immunotherapy, vol. 35, no. 6, pp. 293-299, 2016.

[27] Y. Fujii, M. Kaneko, M. Neyazaki, T. Nogi, Y. Kato, and J. Takagi, "PA tag: A versatile protein tagging system using a super high affinity antibody against a dodecapeptide derived from human podoplanin," Protein Expression and Purification, vol. 95, pp. 240-247, 2014

[28] Y. Maida, M. Yasukawa, N. Okamoto et al., "Involvement of telomerase reverse transcriptase in heterochromatin maintenance," Molecular and Cellular Biology, vol. 34, no. 9, pp. 15761593, 2014

[29] Y. Maid, M. Yasukaw, and K. Masutomi, "De novo RNA synthesis by RNAdependent RNA polymerase activity of telomerase reverse transcriptase," Molecular and Cellular Biology, vol. 36, no. 8, pp. 1248-1259, 2016.

[30] K. Masui, K. Tanaka, D. Akhavan et al., "MTOR complex 2 controls glycolytic metabolism in glioblastoma through FoxO acetylation and upregulation of c-Myc," Cell Metabolism, vol. 18, no. 5, pp. 726-739, 2013.

[31] K. Masui, K. Tanaka, S. Ikegami et al., "Glucose-dependent acetylation of Rictor promotes targeted cancer therapy resistance," Proceedings of the National Acadamy of Sciences of the United States of America, vol. 112, no. 30, pp. 9406-9411, 2015.

[32] C.-K. Park, S.-H. Lee, J. Y. Kim et al., "Expression level of hTERT is regulated by somatic mutation and common single nucleotide polymorphism at promoter region in glioblastoma," Oncotarget , vol. 5, no. 10, pp. 3399-3407, 2014.

[33] T. M. Johanns, Y. Fu, D. K. Kobayashi et al., "High incidence of TERT mutation in brain tumor cell lines," Brain Tumor Pathology, vol. 33, no. 3, pp. 222-227, 2016.

[34] R. J. A. Bell, H. T. Rube, A. Kreig et al., "The transcription factor GABP selectively binds and activates the mutant TERT promoter in cancer," Science, vol. 348, no. 6238, pp. 1036-1039, 2015.

[35] H. Xie, T. Liu, N. Wang et al., "TERT promoter mutations and gene amplification: Promoting TERT expression in merkel cell carcinoma," Oncotarget , vol. 5, no. 20, pp. 10048-10057, 2014.

[36] J. C. Nault, M. Mallet, C. Pilati et al., "High frequency of telomerase reverse-transcriptase promoter somatic mutations in hepatocellular carcinoma and preneoplastic lesions," Nature Communications, vol. 4, p. 2218, 2013.

[37] S. Borah, L. Xi, A. J. Zaug et al., "TERT promoter mutations and telomerase reactivation in urothelial cancer," Science, vol. 347, no. 6225, pp. 1006-1010, 2015.

[38] J. Panero, R. M. Alves-Paiva, A. Roisman et al., "Acquired TERT promoter mutations stimulate TERT transcription in mantle cell lymphoma," American Journal of Hematology, vol. 91, no. 5, pp. 481-485, 2016.

[39] I. Guilleret, P. Yan, F. Grange, R. Braunschweig, F. T. Bosman, and J. Benhattar, "Hypermethylation of the human telomerase catalytic subunit (hTERT) gene correlates with telomerase activity," International Journal of Cancer, vol. 101, no. 4, pp. 335341, 2002. 
[40] H. Noushmehr, D. J. Weisenberger, K. Diefes et al., "Identification of a $\mathrm{CpG}$ island methylator phenotype that defines a distinct subgroup of glioma," Cancer Cell, vol. 17, no. 5, pp. 510$522,2010$.

[41] K. Masui, W. K. Cavenee, and P. S. Mischel, "Cancer metabolism as a central driving force of glioma pathogenesis," Brain Tumor Pathology, vol. 33, no. 3, pp. 161-168, 2016.

[42] C. M. Heaphy, R. F. de Wilde, Y. Jiao et al., "Altered telomeres in tumors with ATRX and DAXX mutations," Science, vol. 333, no. 6041, article 425, 2011.

[43] J. Schwartzentruber, A. Korshunov, X. Y. Liu et al., "Driver mutations in histone $\mathrm{H} 3.3$ and chromatin remodelling genes in paediatric glioblastoma," Nature, vol. 482, pp. 226-231, 2012.

[44] Y. Maida and K. Masutomi, "Telomerase reverse transcriptase moonlights: Therapeutic targets beyond telomerase," Cancer Science, vol. 106, no. 11, pp. 1486-1492, 2015.

[45] S. Yamaguchi, Y. Maida, M. Yasukawa, T. Kato, M. Yoshida, and K. Masutomi, "Eribulin mesylate targets human telomerase reverse transcriptase in ovarian cancer cells," PLOS ONE, vol. 9, no. 11, Article ID el12438, 2014.

[46] O. L. Chinot, W. Wick, W. Mason et al., "Bevacizumab plus radiotherapy-temozolomide for newly diagnosed glioblastoma," The New England Journal of Medicine, vol. 370, no. 8, pp. 709-722, 2014. 


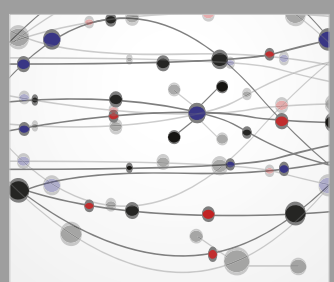

The Scientific World Journal
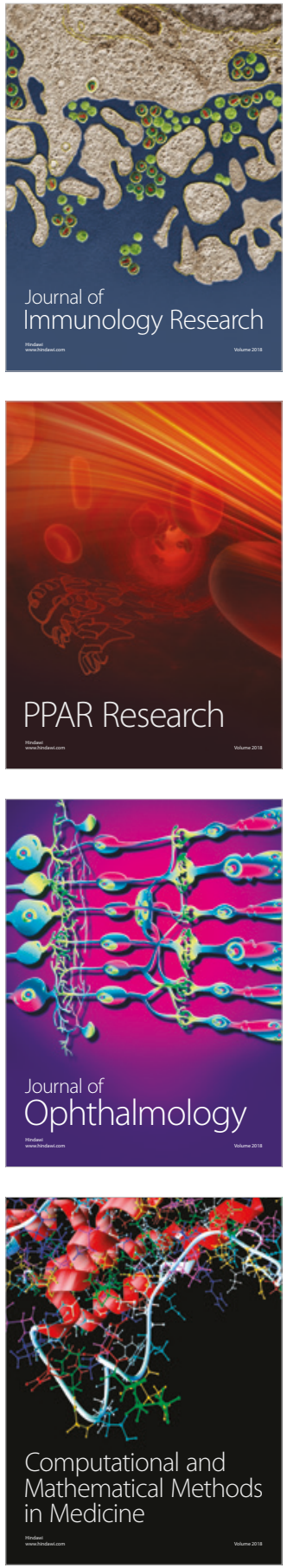

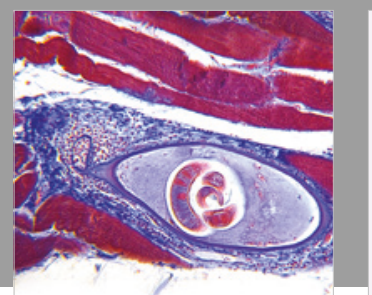

Gastroenterology Research and Practice

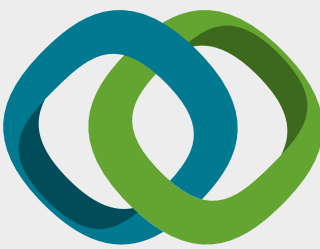

\section{Hindawi}

Submit your manuscripts at

www.hindawi.com
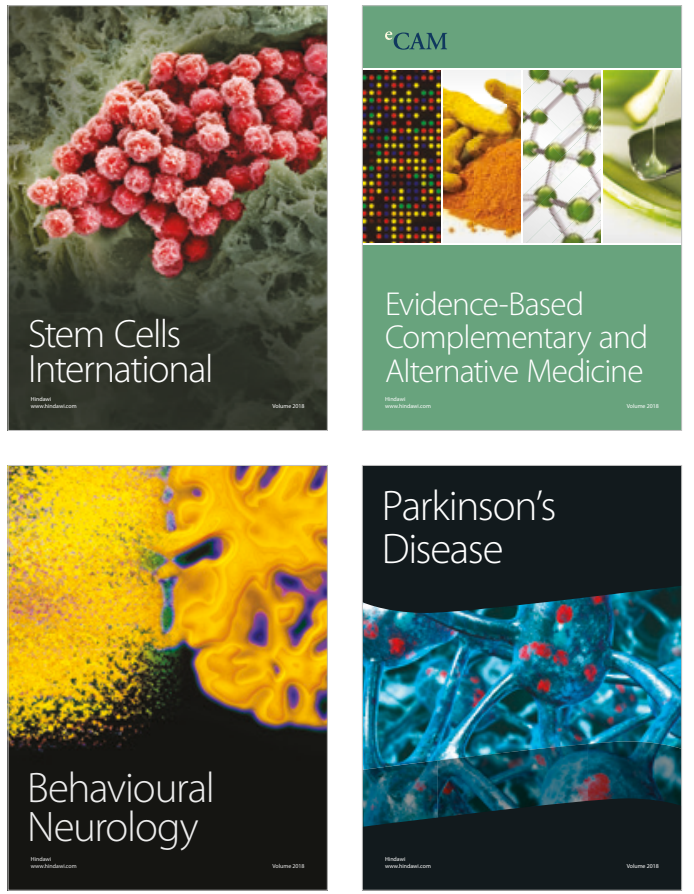

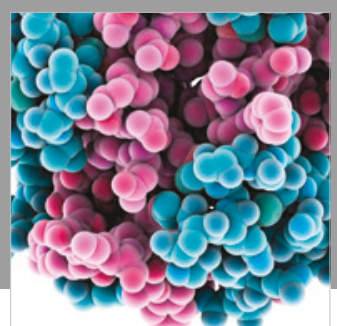

ournal of

Diabetes Research

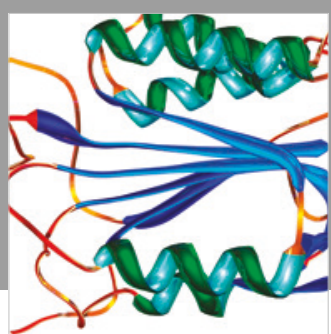

Disease Markers
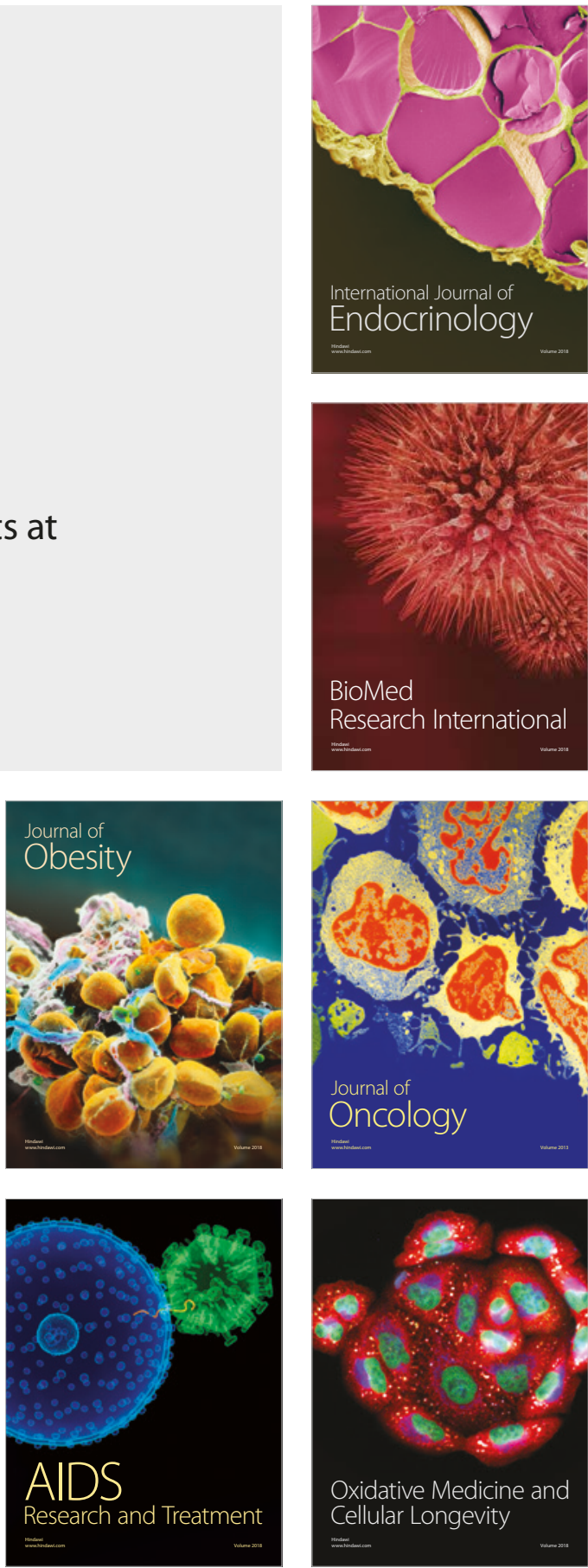